\title{
Comunicação
}

\section{Pegamento e crescimento inicial de mudas de jabuticabeiras 'Açu’ e 'Sabará' submetidas a dois tipos de enxertia}

\author{
Lidiane Rodrigues Londe Franco ${ }^{1}$, Joseilton Faria Silva², Victor Martins Maia ${ }^{3}$, Pollyanna Santiago Lopes ${ }^{2}$, \\ Ismael de Jesus Ferreira Amorim ${ }^{4}$, Edson Hiydu Mizobutsi ${ }^{3}$
}

\section{RESUMO}

A jabuticabeira (Plinia sp.) pertence à família Myrtaceae e é nativa do Centro/Sul/Sudeste do Brasil. O objetivo deste trabalho foi determinar o pegamento e o crescimento inicial de mudas das jabuticabeiras ‘Sabará' e ‘Açu’ submetidas aos métodos de enxertia de garfagem no topo em fenda cheia e garfagem no topo à inglesa simples. Foram avaliados o pegamento inicial aos 50 dias após a enxertia, o pegamento final aos 262 dias após a enxertia, o diâmetro e o comprimento da haste e o número de folhas emitidas aos 78, 107 136, 167, 199, 228 e 262 dias após a enxertia. A cultivar 'Sabará' apresentou maior pegamento inicial quando submetida ao método inglesa simples. A jabuticabeira 'Açu’ obteve maior pegamento final em relação à cultivar 'Sabará'. A cultivar 'Açu’ apresentou maior diâmetro, maior comprimento da haste e maior número de folhas emitidas.

Palavras-Chave: Plinia spp., garfagem, cultivares.

\section{ABSTRACT}

\section{Establishment and growth of grafts of jaboticaba trees 'Açu' and 'Sabara' sumitted to two types of graft}

The jabuticabeira (Plinia sp.) Belongs to the Myrtaceae family and is native to Central / South / Southeast Brazil. The aim of this study was to determining the establishment and growth of grafts of jaboticaba trees 'Sabará' ( $P$. jaboticaba (Vell)) and 'Açu' (P.cauliflora ) submitted to the cleft grafting and whip grafting methods. Were evaluated the initial establishment to 50 days after grafting, the final one at 262 days after grafting, the diameter and lengt of the stem and leaf number appeared to 78, 107 136, 167, 199, 228 e 262 days after grafting. The variety 'Sabará' showed higher initial establishment when subjected to the whip grafting method. The jabuticaba 'Açu' was greater on the final establishment. Cultivar 'Açu’ showed larger diameter, longer length of stem and more leaves.

Key Words: Plinia spp., grafting, variety.

\footnotetext{
Recebido para publicação em dezembro de 2009 e aprovado em maio de 2010

'Engenheira Agrônoma, Mestre. Semiárido da Universidade Estadual de Montes Claros, Caixa Postal 13, 39508-000, Jaíba, Minas Gerais, Brasil. lidiane.agro@yahoo.com.br 2 Engenheiro Agrônomo. Semiárido da Universidade Estadual de Montes Claros, Caixa Postal 13, 39508-000, Jaíba, Minas Gerais, Brasil. joseiltonfs@yahoo.com.br; pollyannasantiago@yahoo.com.br

${ }^{3}$ Engenheiro Agrônomo, Doutor. Departamento de Ciências Exatas e Tecnológicas da Universidade Estadual de Montes Claros, Caixa Postal 91, 39440-000, Janaúba, Minas Gerais, Brasil.victor.maia@unimontes.br; edson.mizobutsi@unimontes.br

${ }^{4}$ Acadêmico do curso de Agronomia. Universidade Estadual de Montes Claros, Caixa Postal 91, 39440-000, Janaúba, Minas Gerais, Brasil. ism.amorim@hotmail.com
} 


\section{INTRODUÇÃO}

Nativa do Brasil a jabuticabeira, (Plinia sp.), encontra-se difundida desde o estado do Pará até ao Rio Grande do Sul, mas é nos estados de São Paulo, Rio de Janeiro, Minas Gerais e Espírito Santo que ocorrem as maiores produções. Pertencente a família Myrtaceae (Mattos, 1983), são conhecidas em torno de 9 espécies de jabuticabeiras (Mattos, 1978), destacando-se as jabuticabas 'Açu' (Plinia cauliflora) e 'Sabará' (Plinia jaboticaba (Vell)), nomenclaturas proposta por Sobral (1985). Ambas produzem frutos apropriados tanto para indústria quanto para consumo in natura. Os frutos possuem elevado valor nutritivo, com altos teores de vitaminas do complexo B, principalmente B2 e niacina, e em menor quantidade a vitamina C, além de sais minerais como ferro, cálcio e fósforo (Mattos, 1983; Donadio, 1983).

Apesar de conhecida há muito tempo e do sabor agradável de seus frutos, a jabuticabeira não tem despertado interesse do fruticultor que a considera inadequada ao cultivo, tendo em vista o longo período juvenil dessa fruteira, que leva aproximadamente dez anos para que uma muda oriunda de sementes comece a produzir (Andrade \& Martins 2003). Segundo Danner et al. (2006), a jabuticabeira leva de oito a quinze anos para que plantas propagadas sexuadamente possam dar início a produção, além disso, a propagação por sementes, possui como desvantagem a segregação genética, originando plantas com grande variabilidade (Fachinello \& Nachtigal, 1992).

Sendo assim, o uso de técnicas de propagação assexuada, que antecipem a produção, além de propiciar maior uniformidade à lavoura, pode contribuir para o desenvolvimento da exploração econômica dessa cultura. Porém, ao contrário de outras frutíferas de clima temperado e subtropical, para a jabuticabeira, ainda não estão estabelecidos métodos eficientes de propagação vegetativa que assegurem a formação de pomares comerciais em curto espaço de tempo (Danner et al., 2006). As plantas enxertadas são mais precoces que as de pé-franco e possuem copas menores, sendo, porém, menos produtivas. Além disso, o valor da muda é consideravelmente elevado (Manica, 2000).

O objetivo do presente trabalho foi determinar o pegamento e o crescimento inicial de mudas das jabuticabeiras 'Sabará' e 'Açu' submetidas aos métodos de enxertia por garfagem de topo em fenda cheia e inglesa simples.

\section{MATERIAL E MÉTODOS}

O experimento foi realizado no viveiro de produção de mudas frutíferas da Universidade Estadual de Montes Claros no Campus de Janaúba, MG, no período de agosto de 2007 a abril 2008.
Os porta-enxertos, usados no experimento, foram oriundos de sementes obtidas a partir de plantas já estabelecidas na região. Após atingirem seis meses de idade, com uma altura variando de 80 a 90 cm e, aproximadamente seis mm de diâmetro, foi realizada a enxertia a uma altura de $25 \mathrm{~cm}$. Os garfos ou enxertos foram provenientes de matrizes de jabuticaba ‘Sabará' e ‘Açu’ da Universidade Federal de Viçosa, MG. Os ramos utilizados com enxertos já estavam bem diferenciados, com uma consistência semilenhosa, comprimento de 10 cm e diâmetro de aproximadamente $6 \mathrm{~mm}$, contendo 6 gemas.

O pegamento inicial foi avaliado, em novembro de 2007, com início das brotações, aos 50 dias após a enxertia. O pegamento final foi avaliado em abril de 2008, aos 262 dias após a enxertia. O número de folhas emitidas, o comprimento e o diâmetro da haste foram avaliados aos 30, 60, 90, 120, 150 e 180 dias após a enxertia.

Observado o pegamento e o desenvolvimento dos enxertos, 70 dias após a enxertia, foram realizadas adubações de cobertura, conforme recomendações de Donadio (2000), tutoramento das mudas em haste única e desbrota constante dos porta-enxertos.

O experimento foi montado segundo o delineamento em blocos casualizados, no esquema fatorial 2 x 2, sendo duas cultivares de jabuticaba 'Sabará' e 'Açu', e dois tipos de enxertia: garfagem no topo em fenda cheia e garfagem no topo à inglesa simples, com oito repetições e quatro plantas por parcela.

Os dados foram submetidos à análise de variância. As médias de pegamento inicial e final obtidas foram comparadas, utilizando-se o teste de Tukey. As avaliações de crescimento foram submetidas à análise de regressão, utilizando-se modelos não lineares. A escolha do modelo de regressão baseou-se no coeficiente de determinação e no potencial para explicar o fenômeno biológico. A análise estatística foi feita com auxílio do Sistema de Análises Estatísticas e Genéticas da Universidade Federal de Viçosa, SAEG V. 5.0 e do Sigma Plot 11.0.

\section{RESULTADOS E DISCUSSÃO}

Houve interação significativa dos fatores estudados apenas para o pegamento inicial. Considerando a jabuticabeira 'Açu', não houve diferença significativa entre os métodos de enxertia testados. Este resultado é semelhante ao encontrado por Araújo \& Castro Neto (2002), trabalhando com diferentes métodos de enxertia em umbuzeiro. Observou-se também que, com exceção da enxertia de garfagem em fenda cheia na cultivar 'Sabará', os demais tratamentos apresentaram valores de pegamentos iniciais estatisticamente iguais e superiores a $90 \%$. O tratamento referente à garfagem em fenda cheia na jabuticabeira 'Sabará' apresentou a menor porcentagem de pegamento inicial com apenas 68,75\% (Tabela 1). 
Tabela 1. Médias da porcentagem de pegamento inicial das jabuticabeiras 'Açu' e 'Sabará' submetidas às enxertias inglesa simples e garfagem em fenda cheia.

\begin{tabular}{lcc}
\hline Método de enxertia / cultivar & 'Açu' & 'Sabará' \\
\hline Inglesa simples & $93,75 \mathrm{aA}$ & $96,88 \mathrm{aA}$ \\
Garfagem fenda cheia & $93,75 \mathrm{aA}$ & $68,75 \mathrm{bB}$ \\
\hline
\end{tabular}

Médias seguidas pela mesma letra minúscula na coluna e maiúscula na linha, não diferem entre si ao nível de 5\% de probabilidade pelo teste de Tukey.

Quanto ao pegamento final houve efeito significativo apenas entre as cultivares. Para esta característica, a cultivar 'Açu' foi superior à 'Sabará', atingindo valores de 87,50\% e 68,75\%, respectivamente (Tabela 2). A média geral dos tratamentos apresentou $78,13 \%$ de pegamento final das mudas. Estes dados indicam que a jabuticabeira 'Açu' apresenta maior potencial para o uso de propagação vegetativa por garfagem em fenda cheia ou em inglêsa simples e provavelmente maior compatibilidade entre o enxerto e o porta-enxerto. O pegamento da cultivar 'Açu' foi bastante satisfatório, próximo a 90\%, permitindo a recomendação dos métodos de propagação estudados para este material. É importante destacar que a adoção desta tecnologia para a jabuticabeira 'Açu' tem o potencial de reduzir significativamente o período entre o plantio e a colheita, além de propiciar a implantação de áreas comerciais uniformes.

Em um estudo realizado por Franzon et al. (2008), utilizando os métodos de enxertia por garfagem em fenda cheia e fenda dupla em pitangueira, o primeiro método apresentou maior valor de porcentagem final de pegamento, que foi avaliada aos 110 dias após a enxertia.

Segundo Manica (2000), trabalhando com jabuticaba, o processo de enxertia de garfagem em fenda cheia resultou em $75 \%$ a $85 \%$ de pegamento dos enxertos. Estes valores foram próximos aos observados para pegamento final e inferiores aos observados para pegamento inicial para a jabuticabeira 'Açu'.

Quanto ao desenvolvimento das mudas, não houve diferença significativa entre os métodos de enxertia utilizados, porém, pode-se observar que a cultivar 'Açu' apresentou uma tendência de crescimento vegetativo mais vigoroso em todas as variáveis avaliadas, confirmando os bons resultados de pegamento desta cultivar.

Tabela 2. Médias da porcentagem de pegamento final das jabuticabeiras 'Açu' e 'Sabará' submetidas aos métodos de enxertia.

\begin{tabular}{lc}
\hline Cultivar & Porcentagem de pegamento final (\%) \\
\hline 'Açu' & $87,50 \mathrm{a}$ \\
'Sabará' & $68,75 \mathrm{~b}$ \\
\hline
\end{tabular}

As médias seguidas pela mesma letra não diferem entre si ao nível de $5 \%$ de probabilidade pelo teste de Tukey.
Em todas as avaliações de crescimento realizadas ao longo do tempo a jabuticabeira 'Açu' foi superior à 'Sabará', confirmando maior vigor e crescimento vegetativo da mesma. Estes dados reafirmam a hipótese baseada nos dados de pegamento incial e final de que a jabuticabeira 'Açu' tem maior potencial para ser indicada para propagação vegetativa por enxertia em garfagem.

Conforme mostrado na figura 1 , a cultivar 'Açu' apresentou diâmetro inicial da haste de $0,89 \mathrm{~cm}$ aos 78 dias após a enxertia e diâmetro final de 4,08 cm aos 262 após a enxertia, valores esses bem superiores aos da cultivar 'Sabará' que teve diâmetro inicial de $0,58 \mathrm{~cm}$ e final de $2,49 \mathrm{~cm}$. Esses valores indicam que a cultivar 'Açu' tem maior compatibilidade com o porta-enxerto utilizado.

A cultivar 'Açu' apresentou comprimento inicial da haste de 5,27 cm, atingindo 25,21 cm aos 262 dias após a enxertia, enquanto que a cultivar 'Sabará', teve comprimento inicial de 3,90 cm e final de 16,18 cm (Figura 2).

Na figura 3, pode-se observar que a cultivar 'Açu' produziu uma média de 6,94 folhas aos 78 dias após a enxertia e, aos 262 dias após a enxertia, o número total foi de 35,28 folhas emitidas. A cultivar 'Sabará' emitiu uma média de 5,83 folhas aos 78 dias após a enxertia e um valor bem menor aos 262 dias, com um total de 25,28 folhas produzidas.

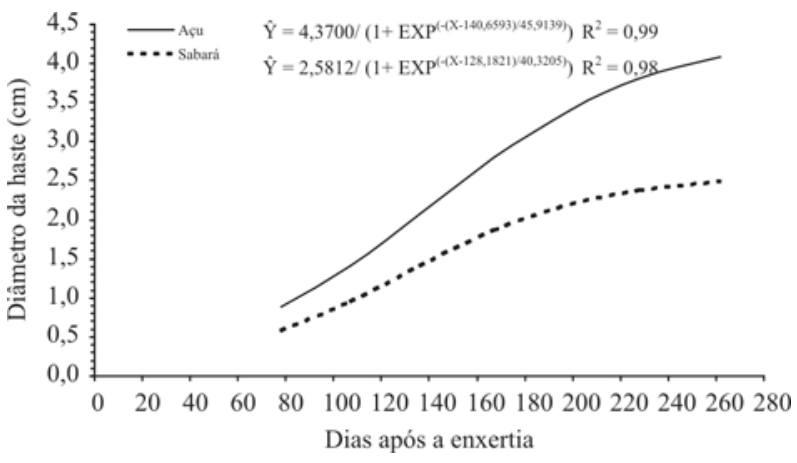

Figura 1 - Diâmetro da haste $(\mathrm{cm})$ das mudas de jabuticabeira das cultivares 'Açu' e 'Sabará' em função de dias após a enxertia.

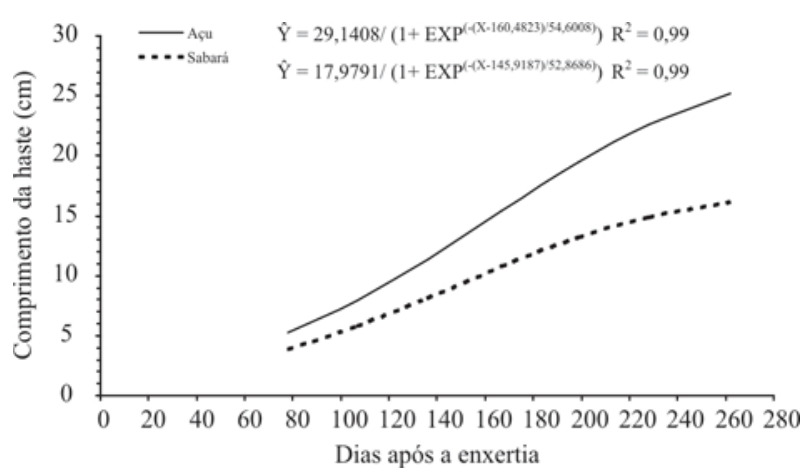

Figura 2 - Comprimento da haste (cm) das mudas de jabuticabeira das cultivares 'Açu' e 'Sabará' em função de dias após a enxertia.

Rev. Ceres, Viçosa, v. 57, n.4, p. 535-538, jul/ago, 2010 


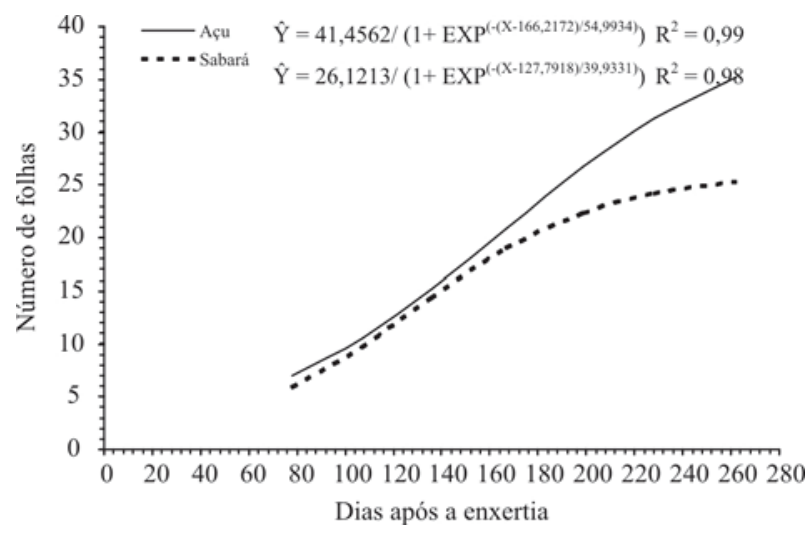

Figura 3 - Número de folhas das mudas de jabuticabeira das cultivares 'Açu' e 'Sabará' em função de dias após a enxertia.

\section{CONCLUSÕES}

A cultivar 'Sabará' apresenta menor pegamento inicial quando submetida a método de garfagem em fenda cheia.

A jabuticabeira 'Açu’ obtém maior pegamento final de enxertia em relação à cultivar 'Sabará' .

Os valores de pegamento de enxertia obtidos neste trabalho permitem indicar o uso desta técnica para propagação assexuada das jabuticabeiras 'Sabará' e 'Açu’.

A cultivar 'Açu' apresentou crescimento vegetativo mais vigoroso das mudas em relação à 'Sabará' .

\section{REFERÊNCIAS}

Andrade RA \& Martins ABG (2003) Influência da temperatura na germinação de sementes de jabuticabeiras. Revista Brasileira de Fruticultura, 25:197-198.

Araujo FP de \& Castro Neto MT de (2002) Influência de fatores fisiológicos de plantas-matrizes e de épocas do ano no pegamento de diferentes métodos de enxertia do umbuzeiro. Revista Brasileira de Fruticultura, 24:752-755. Disponível em <http:// www.scielo.br> Acessado em 20 de março de 2009.

Danner MA, Citadin I, Fernandes Junior AA, Assmann AP, Mazaro SM \& Donazzolo, J (2006) Enraizamento de jabuticabeira (Plinia trunciflora) por mergulhia aérea. Revista Brasileira de Fruticultura, 28:530-532.

Donadio LC (1983) Cuidados com a Jabuticabeira. O Estado de São Paulo, São Paulo. p.16. Suplemento Agrícola

Donadio LC (2000) Jabuticaba (Myrciaria jaboticaba (Vell. Berg). Jaboticabal, Funep. 55p. (Série Frutas Nativas, 3).

Fachinello JC \& Nachtigal JC (1992) Propagação da goiabeira serrana Feijoa sellowiana Berg, através da mergulhia de cepa. Scientia Agricola Piracicaba, 49:p37-39. Disponível em: <http:/ /www.scielo.com.br> Acessado em: 23 de abril de 2009.

Franzon RC, Gonçalves RS, Antunes LEC, Raseira MCB \& Trevisan R (2008) Propagação da pitangueira através da enxertia de garfagem. Revista Brasileira de Fruticultura, 30:488-491.
Manica I (2000) Frutas nativas, silvestres e exóticas 1: técnicas de produção e mercado: abiu, amora-preta, araçá, bacuri, biriba, carambola, cereja-do-rio-grande, jabuticaba. Porto Alegre, Cinco Continentes. 327p.

Mattos JLR (1978) Frutos indígenas comestíveis do Rio Grande do Sul. Porto Alegre, Secretaria da Agricultura. 31p.

Mattos JLR (1983) Frutíferas nativas do Brasil. São Paulo, Nobel. 92p.

Sobral M (1985) Alterações nomeclaturais em plinia (Myrtaceae). Boletim do Museu Botânico de Curitiba, 63:1-4. 